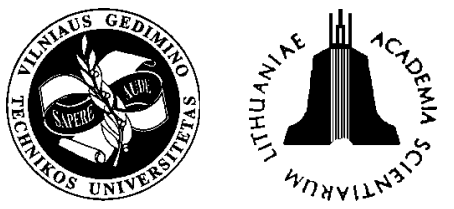

\title{
MATHEMATICAL MODEL OF COMBINED PARAMETRICAL ANALYSIS OF IN INDICATOR PROCESS AND THERMAL LOADING ON THE DIESEL ENGINE PISTON
}

\author{
Sergejus Lebedevas ${ }^{1}$, Galina Lebedeva ${ }^{2}$ \\ ${ }^{1}$ Klaipeda University, Maritime Institute, I. Kanto g. 7, LT-5799 Klaipeda, Lithuania. \\ Telffax: (+370 46 410197), tel:(+37068692086). \\ ${ }^{2}$ Klaipeda University, H. Manto g. 84, LT-5808, Klaipeda, Lithuania. Tel. (+370 61629450). \\ Received 200312 04; accepted 20040217
}

\begin{abstract}
In the publication the methodical aspects of a mathematical model of the combined parametrical analysis of an indicator process and thermal loading on the diesel engine piston have been considered. A thermodynamic model of a diesel engine cycle is developed. The executed development is intended for use during researches and on the initial stages of design work. Its realization for high revolution diesel engines of perspective type CHN15/15 allowed to choose rational variants for the organization of an indicator process and to prove power ranges of application for not cooled and created cooled oil welded pistons.
\end{abstract}

Keywords: diesel engine, indicator process, piston, thermal loading.

\section{Introduction}

The advanced manufacturers of pistons from easy silicon-aluminium alloys (Kolbenschmidt, Mahle etc.) make efforts not only on the expansion of power ranges of piston application(«GalleriKS@», «FibreKS@»models and etc.). The firms realize selective constructive registration and technological consolidation of pistons depending on the level of power, the purpose of diesel engines, and also necessary financial and material investments in the organization of their serial manufacture [1, 2]. For practical realization of such approach it is essentially important to provide the nominate power ranges of application of pistons of various design schemes. It is characteristic that the recommended power ranges of application of pistons of the similar design schemes are widely used. Their range is rather essentially blocked for various types of pistons. This circumstance is treated as a result of the influence of features of an indicator process of a diesel engine. It testifies the proven reserves of the increase of serviceability of the piston at the expense of optimization of parameters of an indicator process.

Therefore, in the context of the power of diesel engines, it is rational to do accent on purposeful formation of power ranges of the application of pistons. Especially it is urgent when the opportunities of the choice of de-

\footnotetext{
${ }^{1}$ E-mail laivum@ji.ku.lt; L_Sergejus@mail.ru

2E-mail galina@ik.ku.lt
}

sign - technological measures of piston local hardening and materials with progressive physical-mechanical properties are limited.

In the executed work such methodical approach is accepted as a basis. It is realized by the development of the combined parametrical analysis of an indicator process, thermal loadings perceived by the piston and limited criteria of thermal stress of the piston. Thus the constructive - technological features of the design of pistons and the used way of cooling are taken into account. The application of development is focused on the stages of design work and outline designing and also on the perfection of parameters of maintained park of diesel engines.

\section{Methodical aspects of a model of the combined parametrical analysis}

The combined parametrical analysis is realized by sharing the generalized dependences of an indicator process and thermal loading on the detail of a cylinder-piston group of a diesel engine.

The generalized dependences of parameters of an indicator process. For the optimization of an indicator process of a diesel engine on the parameters of fuel economy as a basis the known method of search of optimum of an indicator efficiency factor $\eta_{i}$ is accepted. For this purpose generalized interrelations $\eta_{i}$ are used with parameters of an indicator process: factor of surplus of air $(\alpha)$; degree of increase of pressure in the cylinder 
$(\lambda)$; pressure and temperature of air at the beginning of compression in diesel cylinder $\left(P_{a}, T_{a}\right)$ or after turbocompressor $\left(P_{k}, T_{k}\right)$; degree of compression $(\varepsilon)$. The basic advantages of the method consist of the following. The limited number of the independent factors provides the generality of the method which is necessary for the stage of design work. The used graphic form of generalization provides a presentational and clear physical sense of the received results. The analysis is not limited to narrow frameworks of a researched diesel engine complete set (design and adjusting parameters of units). The opportunities of using the experimental data of diesel engines - prototypes are raised by the reliability of the results.

The efficiency of the used method is confirmed by the data of basic researches of Research Institute of Engines (Russia) [3]. Calculated by its authors - professor D. Portnov and S. Pogodin optimum means of $\alpha, \lambda, P_{k}$ and $\varepsilon$ for achievement $\eta_{\text {imax }}$ were successfully used in the work of perfection high revolution transport diesel engines.

Within the framework of the present researches the preference is given to the similar approach of professor N. Ivanchenko and O. Krasovskiy [4]. Rather low dynamics of an indicator process on diesel engines CHN15/15 $(\lambda \approx 1,25 \div 1,35$ against $\lambda \approx 1,8 \div 2,2$ in the works of professor D. Portnov and S. Pogodin is taken into account. Besides the method [4] is more universal. The interrelation of parameters of a cycle is submitted in the form of graphic dependences of complex parameter of level power up $\Pi=\left(P_{\max } /\left(P_{m i}\right)\left(350 / T_{a}\right)\right.$ and $\eta_{\mathrm{i}}$ in the function of $P_{\max } / P_{a}$ (see Fig 1).

The ratio $\mathrm{P}_{\max } / \mathrm{P}_{\mathrm{a}}$, in turn, is a product of two determining parameters of an indicator process. As a result the functional interrelations $\Pi, \eta_{i}=f\left(P_{\max } / P_{a} \alpha\right)$ allow to carry out the multi parametrical analysis of an indicator process depending on: the level of power $\left(P_{m i}\right)$, restrictions of allowable mechanical loadings on details $\left(P_{\max }\right)$ and parameters of air cooling system $\left(T_{a}\right)$. As a result, the optimum $\eta_{i}$ is reached by the choice of rational quantity $\varepsilon$ at accepted $\alpha, P_{\max }, \lambda$ and $P_{k}$.

Parameters of thermal loading on the piston. At the stage of research work and outline designing of diesel engines of unified type, as a rule, it is required to execute a significant number of various estimations of diesel engines parameters. Therefore technically difficult and expensive is the application of experimental and modern settlement methods of research, multi-zone mathematical models and also intensively developing modern integrated systems of computer designing CAD/CAM/CAE/ EPD (Unigraphics, Euclid Quantum etc.) [5].

Their application is more rational at the subsequent stages of design work (technical and work projects). Technically profitable at this stage it is to use the checked in practice methods based on one-zone models. So, in the executed work for account of $\alpha_{G}$ - heat transfer coefficient is applied from a working body (from gas in diesel cylinder) into the bottom of a piston, developed in CNIDI formula (Central Research Diesel Institute, Russia) [6].

The use of $\alpha_{G}$ - formulas in the decision of practical tasks for diesels does not lose urgencies and efficiency $[7,8]$. For example, after the number of modifications known $\alpha_{G}$ - formula of professor G. Woschni is used in researches of both diesel and gas engines [9]. The $\alpha_{G}-$ formula CNIDI can be recognized not less successful. The development is executed on the basis of the experimental researches which have included the definition of

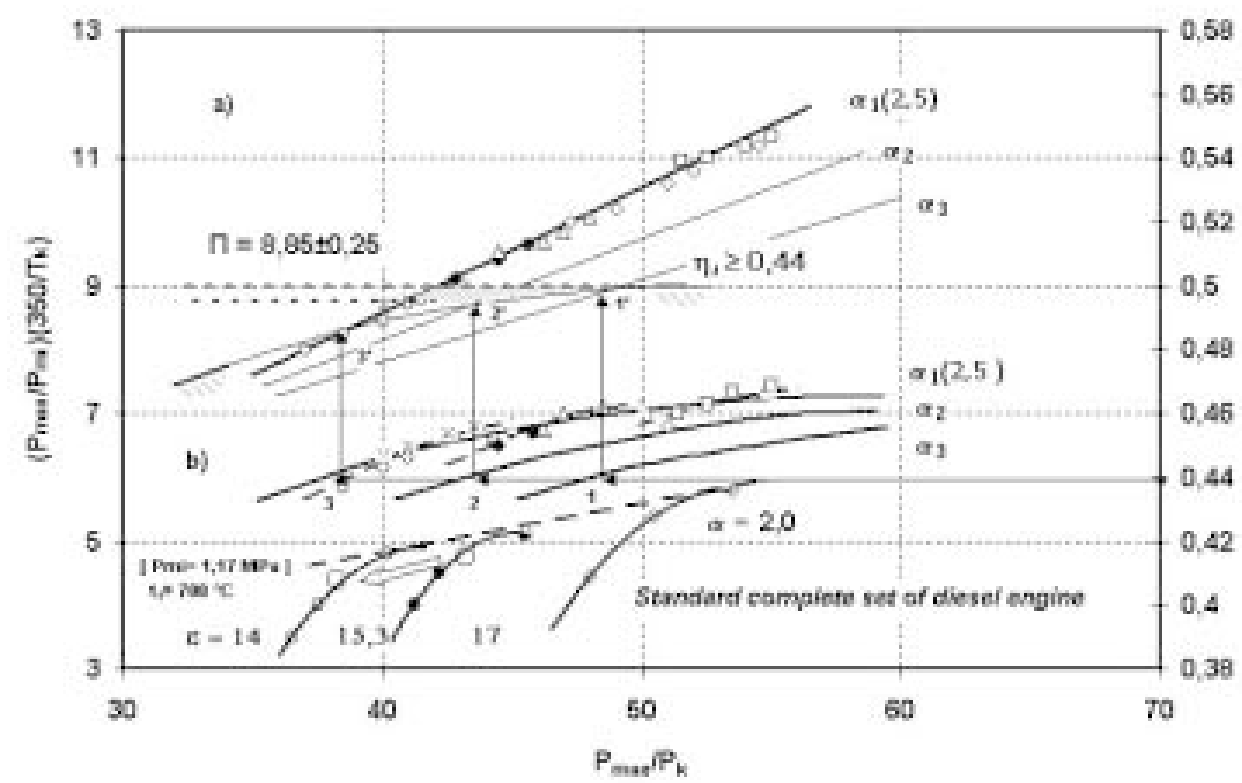

Fig 1. The generalized dependences of parameters of an indicator process of diesel engines CHN15/15:

a) perspective complete set: $\bigcirc, \bullet, \diamond-\varepsilon=14 ; 15,3 ; 17$ without air cooling; $\times, \triangle, \square-\varepsilon=14 ; 15,3 ; 17$ with air cooling; b) serial complete set 
heart balances of diesel engines, registration of parameters of indicator processes and local heart flows in details. As well as in the $\alpha_{G}$ - formula of professor G. Woschni, in the structure of dependence CNIDI criterion function of kind $N u=A P r^{n} R^{m}$ is used. Constants $A, n$, and $\mathrm{m}$ are determined by the generalization of the results of tests of wide scale high and middle revolution diesel engines: 6CHN12/14, 6CHN18/22, 1CHN25/34, 1CHN26/26. Besides their successful approbation for diesel engines CHN5/15, CHN15/15, CHN15/18, CHN16,5/18,5 and CHN16,5/15,5 [10] was taken into account.

Interrelation between parameters of thermal loading on the piston and parameters of an indicator process. The calculation of parameters of heart flow in the combustion chamber component with $\alpha_{G}$ - formula of heart transfer coefficient is based on the use of instant mean mass meanings of temperature $T_{G}$ and pressure $P_{G}$ of a working body in the cylinder, and also its physical characteristics $\left(\lambda_{G}-\right.$ factor of heart conductivity of gas, $\mu_{G}$ - factor of viscosity of gas). The meanings of $\lambda_{G}$ and $\mu_{G}$ depend on $T_{G}, P_{G}$ and the structure of a working body - on a factor of air surplus $(\alpha)$. Therefore the decision of the task is connected with the development of a thermodynamic model of a cycle, which would allow to expect a current of meanings $T_{G}$ in the function of integrated parameters of an indicator process $\left(\alpha, \lambda, \varepsilon, T_{\grave{a}}, P_{a}\right)$.

In this case the combination of parameters of an indicator process is considered as the factor of the form of the cycle indicator diagram or its form-factor.

\section{Thermodynamic model of a cycle of high revolu- tion diesel engine}

The analysis of a thermodynamic model of a diesel engine cycle [10] (further in the text of "cycle") is an expedient to present function $T_{G}=f(\varphi)$, where $\varphi-$ a degree of crank angle $\left({ }^{\circ} \mathrm{CA}\right)$. It is explained by the determining role of the temperature characteristics at the formation of parameters of thermodynamic perfection of a work cycle and thermal loading on the detail of a cylinder - piston group. The substantiation of a cycle model on the combustion - expansion process is executed basing on the data of the references and the results of experimental research of diesel engines (see Fig 2).

Functions $T_{G}=f(\varphi)$ in zone of $T_{\max }$ are characterized by the presence of platform of provisional $T_{\max }$ constancy (isothermal expansion). Its extent changes depend on work regime, adjusting and external parameters of a diesel engine. In particular its extent is increasing at the increase of the injected fuel portion $q_{c i k l}$ (increase $P_{m i}$ ) (see Fig 2a), at the reduction the fuel injection degree in a cylinder $\varphi_{k i}$ (see Fig 2b).

According to the generalized experimental data the part of fuel which is burning down at the end of isothermal expansion, is equal to size $0,7 \div 0,8$. The part of heat transmitted to walls of details of the combustion chamber does not exceed $3 \div 4 \%$. Therefore it is not allocated in the separate component of heat balance.

The accepted thermodynamics of a diesel engine cycle scheme on the site of compression, combustion and expansion are shown in Fig 3.

The thermodynamic cycle $T_{G}=f(\varphi)$ has the number of control points conterminous to actual meanings of the temperature of a working body of a real cycle of a diesel engine. Here $T_{a}, T_{H}, T_{Z}, T_{Y}=T_{T}, T_{b}$ - accordingly is the temperature of the working body at the moment of closing of inlet valves, at the beginning of burning, at the moment of the achievement of its maximal pressure of combustion and maximal temperature of cycle $\left(T_{\max }\right)$, when opening exhaust valves.
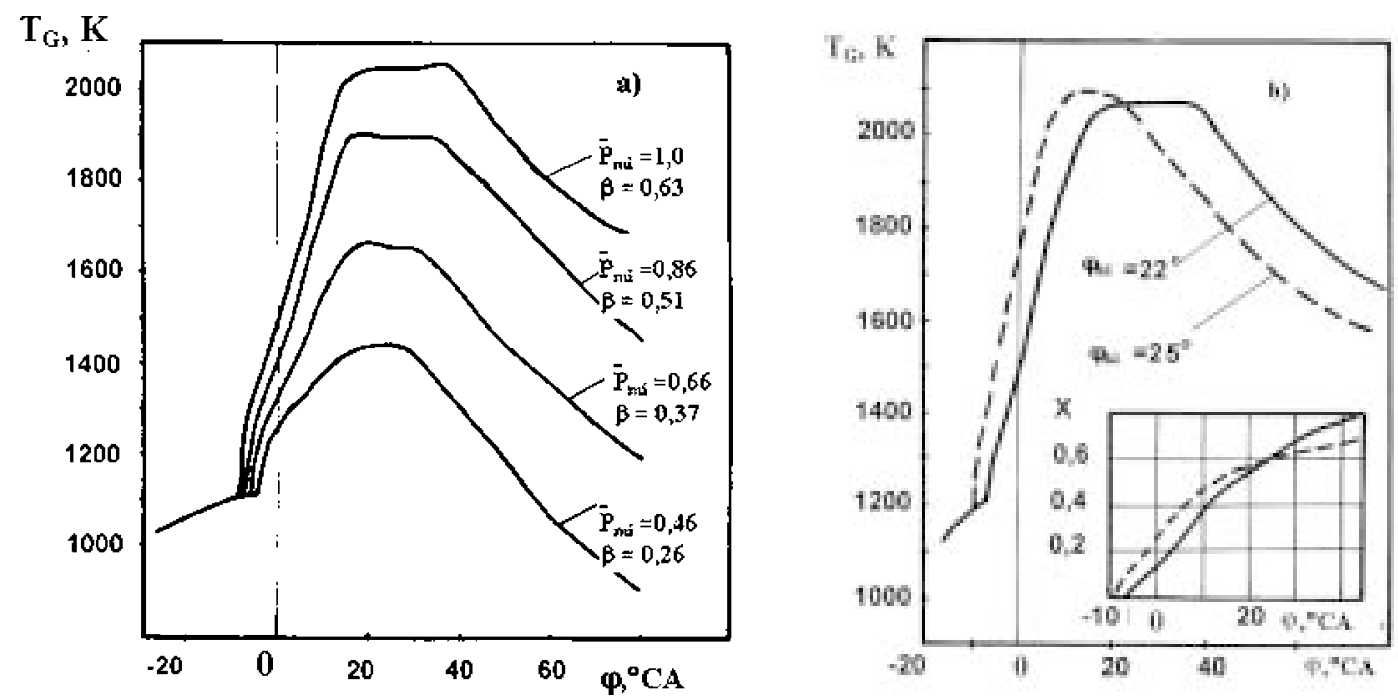

Fig 2. The change of temperature of a working body in the cylinder of a diesel engine: a) CHN15/15; $n=43,3 \mathrm{~s}^{-1} ; P_{\kappa}=0,175 \mathrm{MPa}$;) CHN15/18; $n=33,3 \mathrm{~s}^{-1} ; \beta=0,26 \div 0,63$ 


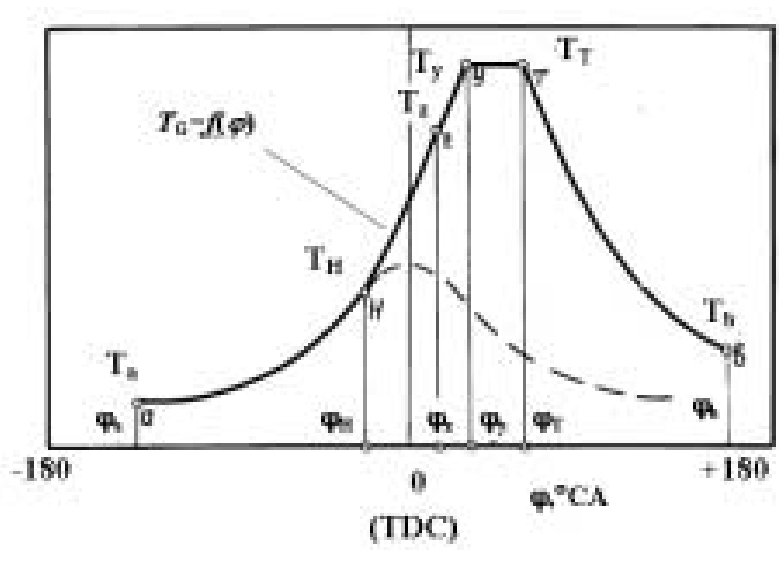

Fig 3. The thermodynamic scheme of a high revolution diesel engine cycle in coordinates $T_{G}-\varphi$

The sites $T_{H}-T_{Y}$ and $T_{Y}-T_{T}$ are described by a linear function $T_{G}=f(\varphi)$. Structure and specific heat of a working body on sites $T_{H}-T_{Z}$ and $T_{Z}-T_{Y}$ are defined on average for these sites by the meanings of temperature and a factor of air using $\beta$ (factor by opposite to factor of surplus of air $-1 / \alpha)$. The mean sizes of parameters of politropes $n_{1}$ and $n_{2}$ for processes of compression from $T_{a}$ to $T_{H}$ and expansion from $T_{T}$ to $T_{b}$ are accepted constant.

The further calculation of a cycle is connected with the establishment of functional dependence between $T_{G}$ and characteristic parameters of cycle, parameters of its dynamics and parts of heat allocated on separate sites of the cycle: $\varphi_{H}-\varphi_{Y} ; \varphi_{Y}-\varphi_{T} ; \varphi_{T}-\varphi_{B}$

Site of compression $\varphi_{\mathrm{a}}-\varphi_{\mathrm{H}}$

Size $T_{\mathrm{G}}=T_{a}\left(\frac{\varepsilon_{\varphi}}{\varepsilon_{a}}\right)^{n_{1}-1}$, where $\varepsilon_{\varphi}=\frac{V_{C}+V_{S}}{V_{C}+V_{\varphi}}$ and $\varepsilon_{a}=\frac{V_{C}+V_{S}}{V_{C}+V_{a}}$ accordingly degree of compression for current $\varphi$ and $\varphi_{\grave{a}} ; V_{C}-$ combustion chamber volume; $V_{S}$ - working volume of the cylinder; $V \varphi$ and $V_{a}$ - volume of the cylinder appropriate $\varphi$ and $\varphi_{\grave{a}}$. Then at the moment of beginning of seen fuel combustion

$$
\left.T_{\mathrm{G}}=T_{a}\left(\frac{\varepsilon}{\varepsilon_{a}}\right)^{n_{1}-1} \text { (here } \varepsilon,=\frac{V_{C}+V_{S}}{V_{C}+V}\right) .
$$

Sites of combustion $\varphi_{\underline{H}}=\varphi_{\underline{Y}}$ and $\varphi_{\underline{Y}}=\varphi_{\underline{T}}$.

It is expedient to enter the concept of temperature analogue of mean rigidity of cycle $\Psi_{1}=\left(T_{Z}-T_{H}\right) /\left(\varphi_{Z}-\varphi_{H}\right)$. In result $T_{G}=T_{H}+\Psi_{1}\left(\varphi-\varphi_{H}\right)$. After representation $V_{H}$ in the function of relative moving of the piston $\bar{S}_{H}$ size $V_{H}=V_{S} \bar{S}$, where $\bar{S}_{H}=S_{H} / S=0,5\left(1+\lambda_{s} / 4-\cos \varphi_{H}-\lambda_{s} /\right.$ $\left.4 \cdot \cos 2 \varphi_{H}\right)$. The trigonometric function $S \varphi=f(\varphi)$ is expedient for presenting $S \varphi=a \varphi^{2}$, where $a=0,228+0,313 \lambda_{s}$, to within $\pm 3 \%$ for ranges $\varphi=0 \div 40^{\circ} \mathrm{CA}$ and $\lambda_{s}$ $=0,23 \div 0,32$ (constant of crankshaft).
Then in the general form

$$
\varepsilon_{\varphi}=\frac{V_{C}+V_{S}}{V_{C}+V_{S} \bar{S}}=\frac{\varepsilon}{1+(\varepsilon-1) a \varphi^{2}}, \text { and for the begin- }
$$

ning combustions $\varphi_{H}$ meaning

$$
\varepsilon_{H}=\frac{\varepsilon}{1+(\varepsilon-1) a \varphi_{H}^{2}} .
$$

With use of the simplified expression for $V \varphi$ and entered $\Psi_{1}$ the calculation pressure

$$
\begin{gathered}
P_{G}=\frac{G_{\mathrm{G}} R_{\mathrm{G}}\left[T_{H}+\Psi_{1}\left(\varphi-\varphi_{H}\right)\right]}{V_{C}+V_{S} a \varphi^{2}}= \\
\frac{G_{\mathrm{G}} R_{\mathrm{G}}(\varepsilon-1)}{V_{S}} \cdot \frac{T_{H}+\Psi_{1}\left(\varphi-\varphi_{H}\right)}{1+(\varepsilon-1) a \varphi^{2}},
\end{gathered}
$$

where $G_{G}$ - mass of a working body; $R_{G}$ - universal gas constant. Here it is expedient from parameter $\Psi_{1}$ to standard parameter of work process - degree of increase of pressure

$$
\lambda=P_{Z} / P_{C}=P_{Z} /\left[P_{H}\left(\varepsilon / \varepsilon_{a}\right)^{n_{1}}\right] .
$$

With the account before the accepted condition of constancy of structure and specific heat of a working body on site $\varphi_{H}-\varphi_{Y}$ is received:

$$
\frac{T_{Z}}{T_{C}}=\frac{P_{Z} V_{Z}}{P_{C} V_{C}}=\lambda \frac{V_{C}+V_{S} a \varphi_{Z}^{2}}{V_{C}}=\lambda\left[1+(\varepsilon-1) a \varphi_{Z}^{2}\right] .
$$

The ratio $\frac{V_{C}+V_{S} a \varphi_{Z}^{2}}{V_{C}}=1+(\varepsilon-1) a \varphi_{Z}^{2}=\rho_{Z}$

will be named as a degree of initial expansion at combustion. After that $T_{Z} / T_{C}=\lambda \rho_{Z}$. After substitution $\Psi_{1}$ in formula (2) and its transformations the ratio of temperatures $T_{Z} / T_{H}$ occurs. For its definition we will address the appropriate pressure at characteristic points of cycle $\lambda_{H}=P_{Z} / P_{H}=\lambda\left(\varepsilon / \varepsilon_{a}\right)^{n_{1}}$.

With the account (1)

$$
\lambda_{H}=\lambda\left[1+(\varepsilon-1) a \varphi_{H}^{2}\right]^{n_{1}}=\lambda \rho_{H}^{n_{1}}
$$

where $\rho_{H}$ - degree of the change of volume of a working body at the initial phase of burning. Then $T_{Z} / T_{H}$ will be expressed by dependence:

$$
\begin{aligned}
& \lambda_{H Z}=\frac{T_{Z}}{T_{H}}=\frac{P_{Z} V_{Z}}{P_{H} V_{H}}= \\
& \lambda_{H} \frac{1+(\varepsilon-1) a \varphi_{Z}^{2}}{1+(\varepsilon-1) a \varphi_{H}^{2}}= \\
& \lambda_{H} \frac{\rho_{Z}}{\rho_{H}}=\lambda \rho_{Z} \rho_{H}^{n_{1}-1},
\end{aligned}
$$


then (2) will be transformed to form:

$P_{G}=\frac{G_{\mathrm{G}} R_{\mathrm{G}}(\varepsilon-1) T_{H}}{V_{S}} \cdot \frac{\frac{\varphi_{Z}-\varphi}{\varphi_{Z}-\varphi_{H}}+\lambda_{H Z} \frac{\varphi-\varphi_{H}}{\varphi_{Z}-\varphi_{H}}}{1+(\varepsilon-1) a \varphi^{2}}$

At the given inclination of straight line $T_{G}=f(\varphi)$ on the first site of heat intake $\left(\Psi_{1}\right)$ angular coordinate of point $\mathrm{Y}$ is defined by ratio $\lambda_{Z Y}=T_{Y} / T_{Z}$ :

$$
\begin{aligned}
& \frac{\varphi_{Y}-\varphi_{H}}{\varphi_{Z}-\varphi_{H}}=\frac{T_{Y}-T_{H}}{T_{Z}-T_{H}}= \\
& \left(\frac{T_{Y}}{T_{Z}}-\frac{T_{H}}{T_{Z}}\right):\left(1-\frac{T_{H}}{T_{Z}}\right)=\frac{\lambda_{Z Y}-\frac{1}{\lambda_{H Z}}}{1-\frac{1}{\lambda_{H Z}}} .
\end{aligned}
$$

Then $\varphi_{Y}=\frac{\lambda_{Z Y} \lambda_{H Z}-1}{\lambda_{H Z}-1}\left(\varphi_{Z}-\varphi_{H}\right)+\varphi_{H}$.

The similar result turns out after transformation

$T_{Y}=T_{Z}+\Psi_{1}\left(\varphi_{Y}-\varphi_{Z}\right)=T_{Z}\left[1+\frac{1+\frac{1}{\lambda_{H Z}}}{\varphi_{Z}-\varphi_{H}}\left(\varphi_{Y}-\varphi_{Z}\right)\right]$.

The heat moved to a working body on I and II sites of the combustion of a cycle is defined as the sum of work and change of internal energy $Q_{Y}=Q_{H Y}{ }^{+}$ $Q_{Y T}=L_{H Y}+\Delta U_{H Y}+L_{Y T}+\Delta U_{Y T}$. Here $Q_{H Y}$ and $Q_{Y T}-$ absolute mean size of heat moved to working body; $L H Y$ and $L Y T$ - work accomplished by work body; $\Delta U_{Y T}$ and $\Delta U_{H Y}$ - change of internal energy of work body.

On the site I of combustion

$$
\begin{aligned}
& \Delta U_{H Y}=G_{\mathrm{G}} c_{V}\left(T_{Y}-T_{Y}\right)=G_{\mathrm{G}} c_{V} T_{Z}\left(\lambda_{Z Y}-\frac{1}{\lambda_{H Z}}\right)= \\
& G_{\mathrm{G}} c_{V} T_{Z}\left(\frac{\lambda_{Z Y} \lambda_{H Z}-1}{\lambda_{H Z}}\right)=G_{\mathrm{G}} c_{V} T_{H}\left(\lambda_{Z Y} \lambda_{Z} \rho_{H}^{n_{1}-1}-1\right),
\end{aligned}
$$

where $c_{V}$ - specific heat of working body under constant volume on site I of combustion. The formula (3) is resulted in kind, more convenient for integration:

$$
\begin{aligned}
& P_{\mathrm{G}}=\frac{G_{\mathrm{G}} R_{\mathrm{G}}(\varepsilon-1) T_{H}}{V_{S}}\left[\frac{\varphi_{Z}-\lambda_{H Z} \varphi_{H}}{\varphi_{Z}-\varphi_{H}} \cdot \frac{1}{1+(\varepsilon-1) a \varphi^{2}}+\right. \\
& \left.\frac{\lambda_{H Z}-1}{\varphi_{Z}-\varphi_{H}} \cdot \frac{\varphi}{1+(\varepsilon-1) a \varphi^{2}}\right] .
\end{aligned}
$$

$$
\begin{aligned}
& \frac{G_{\mathrm{G}} R_{\mathrm{G}}(\varepsilon-1) T_{H}}{V_{S}}=A ; \\
& \frac{\varphi_{Z}-\lambda_{H Z} \varphi_{H}}{\varphi_{Z}-\varphi_{H}}=B ; \\
& \frac{\lambda_{H Z}-1}{\varphi_{Z}-\varphi_{H}}=D ; \\
& \frac{1}{(\varepsilon-1) a}=C^{2},
\end{aligned}
$$

we shall receive:

$$
P_{\mathrm{G}}=A B \frac{C^{2}}{C^{2}+\varphi^{2}}+A D \frac{C^{2} \varphi^{2}}{C^{2}+\varphi^{2}} .
$$

Work on site I of combustion

$$
L_{H Y}=L_{H 0}+L_{0 Y}=-\int_{V_{H}}^{V_{0}} P_{\mathrm{G}} d V+\int_{V_{0}}^{V_{Y}} P_{\mathrm{G}} d V,
$$

where $L_{H 0}-$ work of compression from $\varphi_{I}$ to piston position at the top point; $L_{O Y}-$ work of expansion from piston position at the top dead center (TDC) to $\varphi_{Y}$.

After transition to integration on $\varphi$ with the account $d V=2 a V_{S} \varphi d \varphi$, we receive:

$$
\begin{aligned}
& L_{H Y}=A B \frac{2 V_{S}}{\varepsilon-1}\left(\int_{0}^{-\varphi_{H}} \frac{\varphi}{C^{2}+\varphi^{2}} d \varphi+\int_{0}^{-\varphi_{Y}} \frac{\varphi}{C^{2}+\varphi^{2}} d \varphi\right)+ \\
& A D \frac{2 V_{S}}{\varepsilon-1}\left(\int_{0}^{-\varphi_{H}} \frac{\varphi^{2}}{C^{2}+\varphi^{2}} d \varphi+\int_{0}^{-\varphi_{Y}} \frac{\varphi^{2}}{C^{2}+\varphi^{2}} d \varphi\right) .
\end{aligned}
$$

As a result:

$$
\begin{aligned}
& L_{H 0}=A B \frac{V_{S}}{\varepsilon-1}\left\{\ln \left[\frac{1}{\sqrt{a(\varepsilon-1)}}+\varphi_{H}^{2}\right]-\ln \frac{1}{a(\varepsilon-1)}\right\}+ \\
& A D \frac{2 V_{S}}{\varepsilon-1}\left\{-\varphi_{H}+\frac{1}{\sqrt{a(\varepsilon-1)}} \operatorname{arctg}\left[\varphi_{H} \sqrt{a(\varepsilon-1)}\right]\right\},
\end{aligned}
$$

$$
\begin{aligned}
& L_{0 Y}=A B \frac{V_{S}}{\varepsilon-1}\left\{\ln \left[\frac{1}{\sqrt{a(\varepsilon-1)}}+\varphi_{Y}^{2}\right]-\ln \frac{1}{a(\varepsilon-1)}\right\}+ \\
& A D \frac{2 V_{S}}{\varepsilon-1}\left\{-\varphi_{Y}-\frac{1}{\sqrt{a(\varepsilon-1)}} \operatorname{arctg}\left[\varphi_{Y} \sqrt{a(\varepsilon-1)}\right]\right\} .
\end{aligned}
$$




$$
\begin{aligned}
& L_{H Y}=A B \frac{V_{S}}{\varepsilon-1} \ln \rho_{H} \rho_{Y}+A D \frac{2 V_{S}}{\varepsilon-1}\left(\varphi_{y}-\varphi_{H}\right)- \\
& \left.\frac{1}{\sqrt{a(\varepsilon-1)}}\left\{\operatorname{arctg}\left[\varphi_{y} \sqrt{a(\varepsilon-1)}\right]-\operatorname{arctg}\left[\varphi_{H} \sqrt{a(\varepsilon-1)}\right]\right\}\right\rangle .
\end{aligned}
$$

According to the executed calculations, for $\psi=0 \div 1,0$ within accuracy $\pm 2,5 \%$ we have $\operatorname{arctg} \psi \approx \psi-0,19 \psi^{2}$, where $\psi=\varphi \sqrt{a(\varepsilon-1)}$. After the above-stated replacement

$$
\begin{aligned}
& L_{H Y}=A B \frac{V_{S}}{\varepsilon-1} \ln \rho_{H} \rho_{Y}+ \\
& 0,38 A D \frac{V_{S}}{\varepsilon-1} \sqrt{a(\varepsilon-1)}\left(\varphi_{Y}^{2}-\varphi_{H}^{2}\right) .
\end{aligned}
$$

In the formulas of definition of work $L_{H Y}=L_{I}, L_{Y T}$ $=L_{\mathrm{II}}$ and change of internal energy $\Delta U_{\mathrm{I}}$ and $\Delta U_{\mathrm{II}}$ identical common parameter $G_{G} R_{G} T_{H}$. Therefore it's expedient to enter concept of specific quantity of heat on sites I and II $-\bar{Q}_{I}$ and $\bar{Q}_{I I}$ :

$$
\bar{Q}_{I}=\bar{Q}_{H Y}=\frac{Q_{H Y}}{G_{\mathrm{G}} R_{\mathrm{G}} T_{H}}, \quad \bar{Q}_{I I}=\bar{Q}_{Y T}=\frac{Q_{Y T}}{G_{\mathrm{G}} R_{\mathrm{G}} T_{H}} .
$$

Then, on the site I of combustion

$$
\begin{aligned}
& \bar{Q}_{I}=B \ln \rho_{H} \rho_{Y}+ \\
& 0,38 D \sqrt{a(\varepsilon-1)}\left(\varphi_{Y}^{2}-\varphi_{H}^{2}\right)+\frac{c_{V}}{R_{\mathrm{G}}}\left(\lambda_{H Z} \lambda_{Z Y}-1\right) .
\end{aligned}
$$

For site II of combustion

$$
\begin{aligned}
& \Delta U_{I I}=0, \\
& L_{I I}=G_{\mathrm{G}} R_{\mathrm{G}} T_{Y} \ln \frac{V_{T}}{V_{Y}}=G_{\mathrm{G}} R_{\mathrm{G}} T_{Y} \ln \frac{\rho_{T}}{\rho_{Y}}, \\
& \bar{Q}_{I I}=\lambda_{H Z} \lambda_{Z Y} \ln \frac{\rho_{T}}{\rho_{Y}},
\end{aligned}
$$

where $\rho_{Y}=\frac{V_{C}+V_{S} a \varphi_{Y}^{2}}{V_{C}}-$ degree of expansion of a working body on site I of combustion at point $Y$; $\rho_{T}=\frac{V_{C}+V_{S} a \varphi_{T}^{2}}{V_{C}}-$ degree of expansion of working body on site II of combustion at point $T$.

If to use $\rho_{Y}=1+a(\varepsilon-1) \varphi_{Y}{ }^{2}$ and $\rho_{T}=1+a(\varepsilon-1) \varphi_{T}^{2}$, we receive $\varphi_{Y}^{2}-\varphi_{H}^{2}=\frac{\rho_{Y}-\rho_{H}}{a(\varepsilon-1)}$.

After transformation

$$
\begin{aligned}
& \bar{Q}_{I-I I}=\bar{Q}_{I}+\bar{Q}_{I I}=B \ln \rho_{Y} \rho_{H}+ \\
& 0,38 D \frac{\rho_{Y}-\rho_{H}}{\sqrt{a(\varepsilon-1)}}+\lambda_{H Z} \lambda_{Z Y} \frac{\rho_{T}}{\rho_{Y}}+\frac{c_{V}}{R_{\partial}}\left(\lambda_{H Z} \lambda_{Z Y}-1\right) .
\end{aligned}
$$

The left part of the expression (5) in view of the accepted condition $Q_{\mathrm{I}-\mathrm{II}}=(0,7 \ldots 0,8) G_{f} Q_{H}$, and also $\beta=$ $14,3 G / G_{a i \tilde{a}}$ will be transformed to:

$$
\bar{Q}_{I-I I}=\frac{(0,7 \ldots 0,8) Q_{H}}{(1+14,3 / \beta) T_{H} R_{\mathrm{G}}}
$$

Site of combustion $\varphi_{T}-\varphi_{b}$

For the definition of $n_{2}$ we shall take advantage of the known equation of balance of heat moved to a working body during politropic expansion

$$
Q_{T b}=\frac{k-n_{2}}{n_{2}-1}\left(c_{V T} T_{T}-c_{V b} T_{b}\right) G_{\mathrm{G}}(k-\text { parameter of }
$$

adiabate; $c_{V T}$ and $c_{V b}-$ specific heat of a working body under constant volume at point $T$ and point $b$ ). In this case $Q_{T b}$ corresponds to active allocation of heat $(\xi)$ on the site $\varphi_{T}-\varphi_{b}$ and calculate on the equation

$$
Q_{T b}=Q_{H} G_{f}\left(\xi_{b}-\xi_{T}\right)=Q_{H} G_{f} \Delta \xi_{T b}
$$

Then

$$
Q_{H} G_{f} \Delta \xi_{T b}=\frac{k-n_{2}}{n_{2}-1}\left(c_{V T} T_{T}-c_{v B} T_{b}\right) G_{\mathrm{G}}
$$

or

$$
Q_{H} \frac{\beta}{14,3+\beta} \Delta \xi_{T B}=\frac{k-n_{2}}{n_{2}-1}\left(c_{V T} T_{T}-c_{V b} T_{b}\right) .
$$

Having designated the left part of equality (7) by factor $a$ and parameter in brackets of the right part $-b$, we shall receive $n_{2}=\frac{k+a / b}{a / b+1}$.

The mean size $n_{2}$ is defined on the equation (8) by the method of iterations.

The developed algorithm allows to simulate $T_{G}$ and $P_{G}$ in function $\varphi$ on site of compression - combustion expansion of cycle of high revolution diesel engine depending on $\beta, \lambda, \lambda_{Z Y}, T_{a}, T_{b}, \varepsilon, \varphi_{H}, \varphi_{Z}$. The subsequent analysis has allowed to establish rather small influence of $\lambda_{Z Y}, \varphi_{H}$ and $\varphi_{Z}$ on $T_{G}$ and to reduce number of independent arguments to $\beta(\alpha), \lambda, \varepsilon, T_{a}$.

As an example in Fig 4 the dependences $T_{G}=f(\varphi)$, designed are given on the basis of the real indicator diagrams and developed algorithm. 

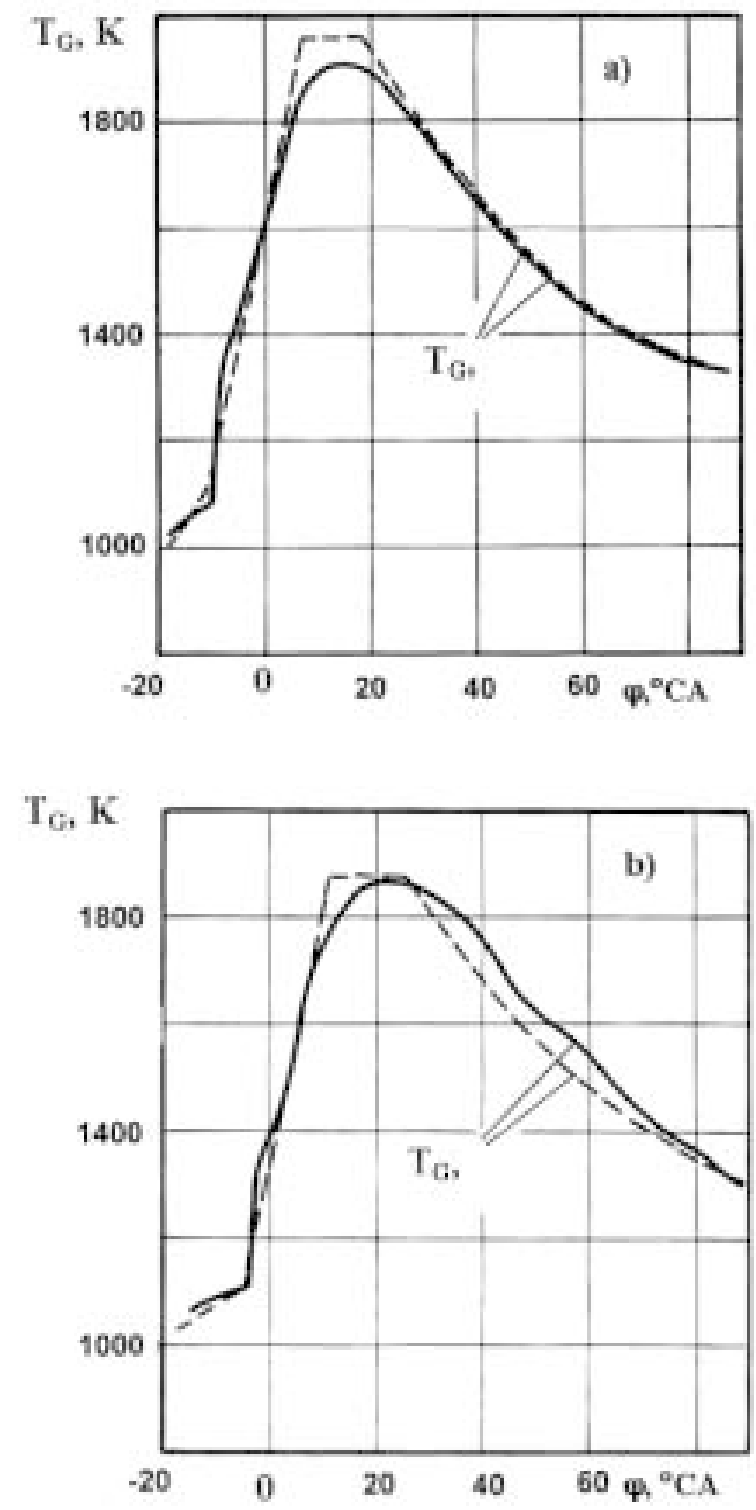

Fig 4. Comparison of the current meanings of a working body temperature in the cylinder of a diesel engine 12CHN15/18: a) $n=33,3 \mathrm{~s}^{-1} ; \varphi_{k i}=25^{\circ} \mathrm{CA}$ before TDC; b) $\mathrm{n}=33,3 \mathrm{~s}^{-1} ; \varphi_{k i}=22{ }^{\circ} \mathrm{CA}$ before TDC; experiment, - - - - calculation

\section{Parameters of an indicator process and thermal loading on the piston}

Definition and research of interrelation mean for a cycle (mean of heart transfer process) quantity of $\alpha_{G . m}$, $T_{G . m}\left(\alpha^{D E T}{ }_{\text {G.m.H }}, \mathrm{T}^{D E T}{ }_{\text {G.m.H }}\right)$ with parameters of an indicator process is based on the research [10]. Its basis is the mathematical model of cycle high revolutions diesel engine which is intended for the calculation of the parameters of a working body and the characteristics of heart formation $T_{G}, P_{G}, X=f\left(\alpha, \varepsilon, \lambda, P_{a}, T_{a}\right)$. The coefficient of heart transfer from a working body in to the bottom of a piston [6] is defined by the equation:

$$
\begin{aligned}
& \alpha_{G}=\left(2,75+58,6 \frac{D}{C_{m}}\right) \times \\
& \frac{\lambda_{G}}{\mu_{G}^{0,5}}\left(\frac{C_{m}}{D}\right)^{0,5}\left(\frac{P_{G}}{R_{G} T_{G}}\right)^{0,5} \mathrm{~W} /\left(\mathrm{m}^{2} \cdot \mathrm{K}\right),
\end{aligned}
$$

$\lambda_{G}$ - factor of heart conductivity of gas, $\mathrm{W} /(\mathrm{m} \cdot \mathrm{K}) ; \mu_{G}-$ factor of viscosity of gas, $\mathrm{kg} /(\mathrm{m} \cdot \mathrm{s}) ; P_{G}$ and $T_{G}-$ accordingly, pressure and temperature of gas, $\mathrm{N} / \mathrm{m}^{2}$ and $\mathrm{K} ; C_{m}$ - mean speed of the piston, $\mathrm{m} / \mathrm{s} ; D$ - diameter of the cylinder, $\mathrm{m} ; R_{G}-$ gas constant, $\mathrm{J} /(\mathrm{kg} \cdot \mathrm{K})$.

For structurally similar diesel engines factor $\left(2,75+58,6 \frac{D}{C_{m}}\right)\left(\frac{C_{m}}{D}\right)^{0,5}$ is entered in the category of constants $(N)$. After that (9) will be transformed to $\alpha_{G}=N \frac{\lambda_{G}}{\mu_{G}^{0,5}}\left(\frac{P_{G}}{R_{G} T_{G}}\right)^{0,5}$. The transition to the combined parametrical analysis is reflected by a system of the equations (10)-(12):

$$
\begin{aligned}
& \alpha_{G}=N \frac{\lambda_{G}}{\mu_{G}^{0,5}}\left(\frac{P_{G}}{R_{G} T_{G}}\right)^{0,5}, \\
& \lambda_{G}, \mu_{G}=f\left(\alpha, X, T_{G}\right), \\
& T_{G}, P_{G}, X=f\left(\alpha, \varepsilon, \lambda, P_{a}, T_{a}\right) .
\end{aligned}
$$

At the joint decision (10)-(12) the conditions of similarity for parameters of heart transfer from a working body into the bottom of a piston are expressed by dependence $\alpha_{G}, T_{G}=f\left(\varepsilon, \alpha, \lambda, P_{k}, T_{k}\right.$ ) (without an appreciable error for calculations it is accepted $P_{k} \approx P_{a}$, $T_{k} \approx T_{a}$ ). The block-scheme of a model of the combined parametrical analysis is given in Fig 5.

The transition to the average for cycle $\alpha_{G . m}, T_{G . m}$ $\left(\alpha_{\text {G.m.H }}^{D E T}, T_{\text {G.m.H }}^{D E T}\right)$ is based on the statistical analysis of results of multi-alternative accounts. The definition of the most important factors of regress in polynomial dependences $\alpha_{\text {G.m }}$ and $T_{\text {G.m }}$ is executed by the analysis of the saved sum of squares at the expense of regress $Q_{p}$ and criterion of Fisher $F_{\alpha, n 1, n 2}$. It is known that the increase of a number of the factors (parameters of a cycle) worsens the degree of conditionality of matrix of normal levels and results in the deterioration of stability of a regress model. The introduction of the most important factor results in sharp change of $Q_{p}$ and $F_{\alpha, n 1, n 2}$. The process of input can be stopped, if the next factor brings $Q_{p}$ share smaller than established beforehand. In the present researches $2,5 \%$ was accepted. 


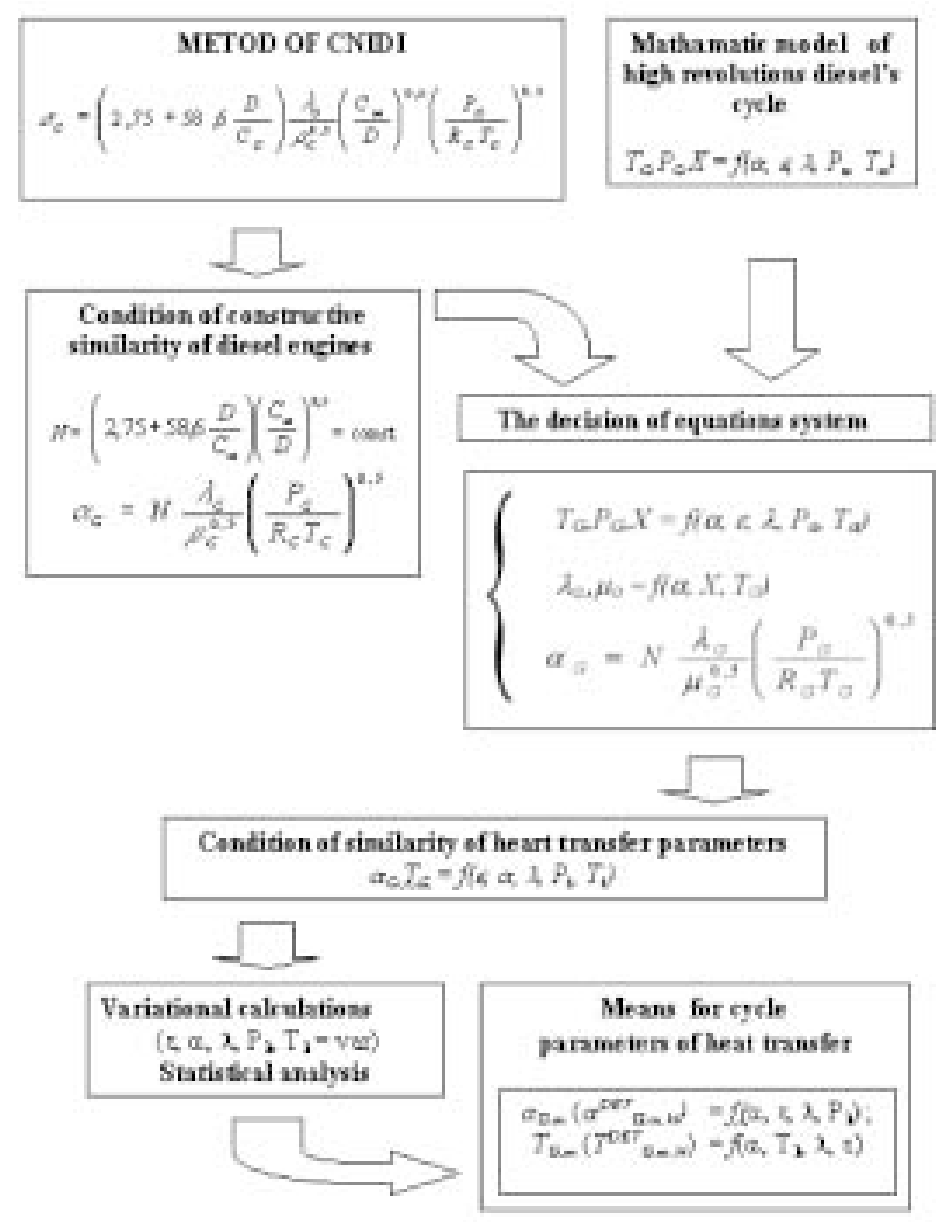

Fig 5. The block- scheme of the combined parametrical analysis

As a result it is determined that the most important factors located on the degree of influence on $\alpha_{\mathrm{G} . \mathrm{m}}, T_{G . m}$, are: for $\alpha_{\text {G.m }}\left(\alpha^{D E T}{ }_{\text {G.m.H }}\right)-\alpha, \varepsilon, \lambda, P_{k}$; for $T_{\text {G.m }}\left(T_{\text {G.m.H }}^{D E T}\right)$ $-\alpha, T_{k}, \lambda, \varepsilon$. For the convenience of practical use the dependences $\alpha_{\text {G.m }}\left(\alpha^{D E T}{ }_{\text {G.m.H }}\right)$ and $T_{\text {G.m }}\left(T_{\text {G.m.H }}^{D E T}\right)$ are submitted in function of the interconnected parameters of a diesel engine $P_{\max } / P_{k}, \alpha, \varepsilon, \lambda, T_{k}$. The influence of parameters of air after turbocompressor $\left(P_{k}\right.$ and $\left.T_{k}\right)$ is reflected in the form of additional factor - accordingly $\alpha_{\text {G.m.H }}^{D E T} / P_{k}^{0.5}=f\left(P_{\max } / P_{k}, \alpha\right)$ and $T^{D E T}{ }_{\text {G.m.H }}\left(T_{k} / 350\right)^{0.5}$ $=f\left(P_{\max } / P_{k}, \alpha, \varepsilon\right)$. Thus, the necessary conditions for the performance of the combined parametrical analysis of the characteristics of an indicator process and heart mechanical intensity of a piston in function $P_{\max } / P_{k}$, and $\varepsilon$ or $\varepsilon, \alpha, \lambda, P_{k}$ and $T_{k}$, taking into account $P_{\max } / P_{k}=\varepsilon^{n} \lambda$ are executed.

\section{Combined parametrical analysis of an indicator process and thermal loading on the piston}

The feature realization of the above-stated method consists of simultaneous scope by the analysis of all researched range of power of diesel engines type on $P_{m i}$ [11]. Such approach answers the basic condition of unification of diesel engines of a unified standard type. It creates the preconditions to the development of uniform principles of a realized indicator process and allows in full to estimate the efficiency of the accepted design decisions on the acquisition of models of a type (first of all by units of fuel and maintenance by air systems). There are investigated levels of perspective power up $P_{m i}=1,2$; 1,$4 ; 1,6 \mathrm{MPa}$ in the executed work. Using the generalized interrelations of parameters of an indicator process $\eta_{i}$, $P_{\max } /\left(P_{m i} T_{k}\right)=f\left(\varepsilon, \alpha, P_{\max } / P_{k}\right)$ the possible variants of organization of an indicator process are determined. The variants are considered as combinations of parameters $\alpha, P_{a}, \lambda, \varepsilon$ (4 combinations a number of the fixed levels $\alpha=$ const). The restrictions $P_{\text {max }}$ and a chosen variant of air cooling system (ACS) on condition $T_{a}=$ const are taken into account. The task of extreme allowable meaning $P_{\text {max }}$ took into account real safety factors of details of diesel engines. At the choice of the combi- 
nations of parameters of an indicator process, the condition of approximate constancy of the specific effective fuel consumption $b_{e} \approx$ const was realized.

For each of the considered combinations of the parameters of a diesel engine the meanings $\alpha^{D E T}{ }_{\text {G.m.H }}$ and $T^{D E T}{ }_{\text {G.m.H }}$ were calculated. Then their combinations as points are put in a nomogram field $\alpha^{D E T}{ }_{\text {G.m.H }}-T^{D E T}{ }_{\text {G.m.H }}$ (see Fig 6). To each of the analysed levels of power $P_{m i}$ in a nomogram field there are equivalens as the local area is limited to the appropriate combinations $\alpha^{D E T}$ G.m.H and $T^{D E T}$ G.m.H.

For the parameters of fuel economy all combinations $\alpha, P_{k}, \lambda, \varepsilon$ are invariant: for fixed $P_{m i}$ its realized condition $b_{i}=$ const, and in all range $P_{m i}=1,2 \div 1,6 \mathrm{MPa}-$ condition $b_{e} \approx$ const. In a nomogram field the parameters of thermal intensity are reflected, which are limited to the reliable work of the researched design of a piston (maximal temperature of piston bottom, temperature of characteristic zones of design $T_{D E T}$, stress $\sigma_{D E T}$ ). The limited parameters of thermal intensity are reflected in the form of lines of constant levels. The introduction in the analysis of several criteria allows to allocate the limiting and to concentrate attention on them. In the considered case in Fig 6 reliability of work of a piston is connected with the level of characteristic temperature in a zone first ring $t_{P r 1}=220^{\circ} \mathrm{C}$.

The developed approach is not limited to the analysis of stationary loading on the piston. The analysis of design of the piston is possible using the criterion of fatiqued durability. Arising stress from the stationary temperature field is taken into account and also variable mechanical loadings (from influence $P_{\max }$ ). Here it is true to use the results fatiqued of tests of the piston and to generalize them in the form of the diagram of Gudman - Serensen.

The analysis of a mutual rule of lines of constant levels $T_{D E T}, \sigma_{D E T}=$ const and fields of researched levels of power up $P_{m i}$ allows to make the multi alternative estimations of rational organization of an indicator process. As a result the application of not cooled piston or the necessity of use of the cooled piston is proved.

For example, for CHN15/15 at equal thermal loading of the piston the reserve of power on $P_{m i}$ in comparison with a base complete set of a diesel engine makes about $20 \%: 1,4 \mathrm{MPa}$ against $1,15 \mathrm{MPa}$. The mutual rule of fields and levels perspective $(\bigcirc)$, and realized $(\mathbf{O})$ of power up in Fig 6 testifies it. The prevailing influence on the temperature of a piston is rendered by $\alpha$ mean. Theoretically, the increase of $\alpha$ from 1,75 to 2,25 keeps constant thermal loading on the piston (modes I and IIIII). Therefore, it is possible to use not cooled piston. In real conditions the maintenance of the reliable starting characteristics of diesel engines limits the borders of possible decrease $\varepsilon$ to $13 \div 14$. The range of possible change $\alpha$ is accordingly narrowed also. Otherwise in view of low dynamics of an indicator process the condition on restriction $P_{\text {max }}$ is not satisfied. As a result, the transition to cooled by oil piston becomes compelled already from $P_{m i} \geq 1,2 \mathrm{MPa}$.

Similarly there are decisions of tasks for other variants of a complete set of diesel engines. On the basis of the offered combined parametrical analysis method the complex of the computer programs was created and successfully applied in practice. In particular, using the electronic - beam welding and hardening in vacuum

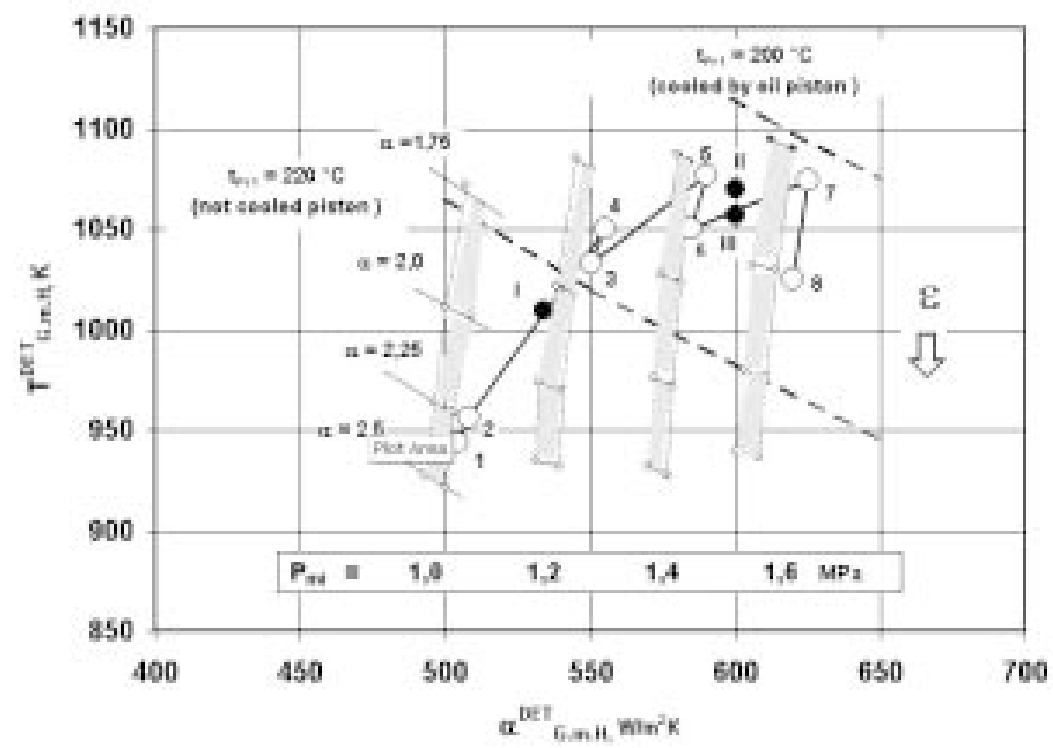

Fig 6. The combined parametrical analysis of an indicator process and thermal loading on a piston of diesel engines type CHN15/15: $\bigcirc$ - increased fuel injection pressure, $\bullet$ - standard complete set ( I- $\varepsilon=15,3 ; P_{m i}=0,96 \mathrm{MPa}$, II $-\varepsilon=15,3$; $\left.P_{m i}=1,17 \mathrm{MPa}, \mathrm{III}-\varepsilon=14 ; P_{m i}=1,17 \mathrm{MPa}\right)$ 
the welded cooled by oil piston is created for diesels CHN15/15. It is supplied with the optimum temperature of zone of the first ring and the mechanical stress in basic elements of a piston are reduced by $30 \%$. The modern computer software is applied for settlement research: 3D designing of details and units "SolidWorks" and mathematical modelling of temperature fields, stress and deformations of details "CosmosWorks". The creation of a volumetric certainly - element model of the piston allowed to combine the research of the temperature and intense condition of the piston. The volumetric certainly - element model of the piston and the received results are reflected in Table and Fig 7.

\section{Conclusions}

The complex of theoretical, calculation, design and experimental research which allowed to develop and realize in practice the complex approach to the creation of pistons for high revolutions diesel engines is executed. The methodical basis of the development is based on the use of the combined parametrical analysis of an indica- tor process and thermal loading on the piston. Thermodynamic model of a diesel engine cycle is developed, which would allow to expect a current of meanings $T_{G}$ in function of integrated parameters of an indicator process $\left(\alpha, \lambda, \varepsilon, T_{a}, P_{a}\right)$.

The practical realization of the method is successfully executed for diesel engines of perspective type CHN15/ 15: their power was on $60 \div 70 \%$ to level $P_{m i}=1,6 \mathrm{MPa}$.

The developed method is realized on the basis of the principle of influence on thermal loading of a piston (details of a cylinder - piston group). It allows to expand the potential use of various constructive complete sets of piston expanding their power ranges of application on $P_{m i}$.

For perspective standard type of diesel engines CHN15/15 the range of use of the not cooled piston is extended to $30 \div 40 \%$. The positive results of the approbation of the method for high revolution diesel engines of perspective standard type CHN15/15, CHN15/18, CHN16,5/18,5 testify its profitability. The method is expedient for use during research design work and outline design - at a stage when the shape of a product and its basic design data are formed.

Temperature of the welded piston of diesel engines CHN15/15 $\left(P_{m i}=1,6 \mathrm{MPa}\right)$

\begin{tabular}{|l|l|l|l|l|l|}
\hline \multirow{2}{*}{ Complete set of piston } & \multicolumn{5}{|c|}{ Temperature of the piston, ${ }^{\circ} \mathrm{C}$} \\
\cline { 2 - 7 } & $\mathrm{t}_{P c}$ & $t_{P \max }$ & $t_{P p}$ & $t_{P r 1}$ & $t_{P r 2}$ \\
\hline Not cooled piston & 280 & 285 & 272 & 240 & 202 \\
\hline Cooled piston (circulation) & 250 & 255 & 243 & 205 & 162 \\
\hline Cooled piston (shaking) & 238 & 239 & 227 & 190 & 157 \\
\hline
\end{tabular}
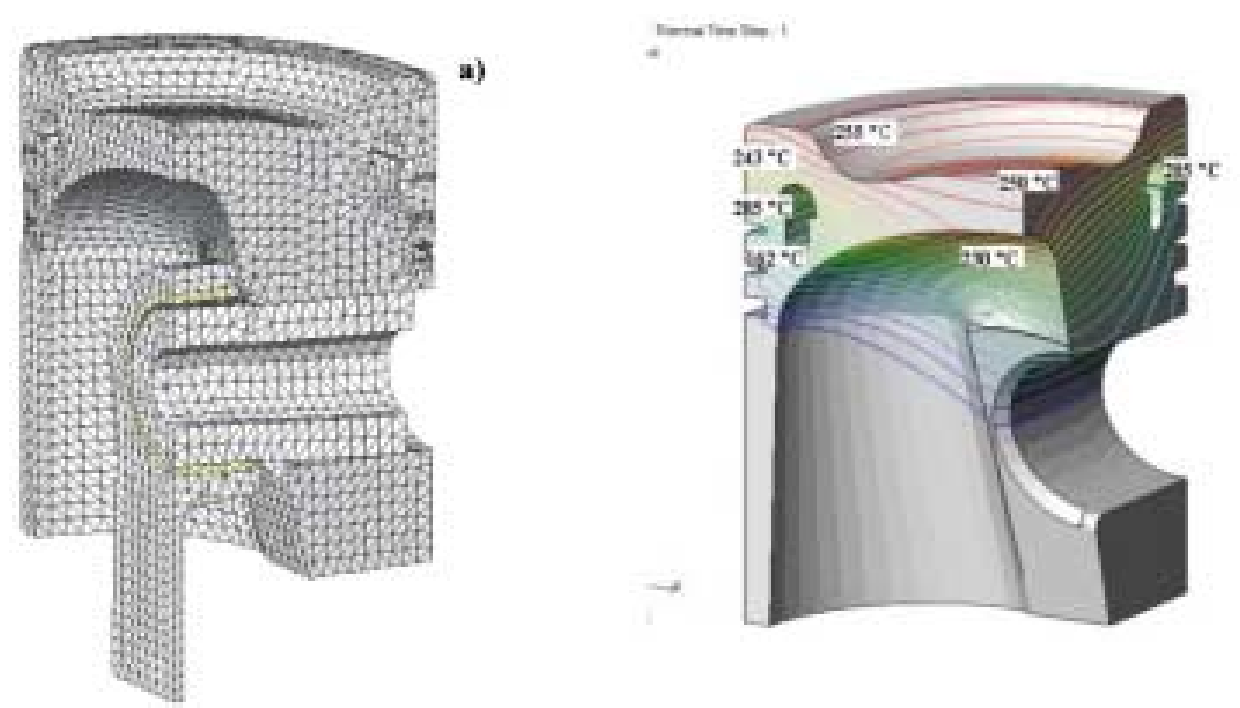

b)

a)

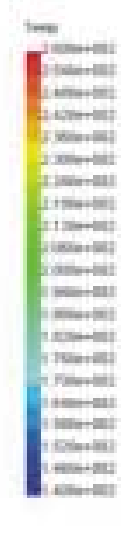

Fig 7. The volumetric certainly - element model of the piston: a) and temperature field, b) of the welded cooled piston of diesel engines CHN5/15 (experimental compete set, $P_{m i}=1,6 \mathrm{MPa}, n=2600 \mathrm{~min}^{-1}$ ) 


\section{References}

1. Pistons for Passenger Car Diesel Engines. Pistons for Commercial Vehicle Applications (on materials of firm Mahle), 2002.

2. Piston technology for passenger car / Diesel engines: Maximum power density with KS pistons (on materials of firm Kolbenschmidt), 2002.

3. Portnov D. A. High-speed turbo-piston engines with ignition from compression. Moscow: Mashinostroeniye, 1963, $638 \mathrm{p}$.

4. Ivanchenko N. N., Krasovskij O. G., Sokolov S. S. High supercharge of diesel engines. Moscow: Mashinostroeniye, 1982. $198 \mathrm{p}$.

5. Jolgaf M. and etc. Development of a CAD/CAM system for the closed-die forging process. M. Jolgaf, A. M. S. Hamouda, S. Sulaiman and M. M. Hamdan. Journal of Materials Processing Technology, Vol 138, Issues 1-3, 20 July 2003, p. 436-44.

6. Molodcov N. I. and etc. Experimental installations, methods and results of heart technical researches of parameters of diesel engine. In: N. I. Molodcov, V. P. Sokolov, M. L. Chirin. Works of CNIDI, 69, 1975, p. 33-54.

7. Kavtaradze R. The local heat exchange in piston engines. The N. E. Bauman's Moscow State University. Moscow, 2001. $592 \mathrm{p}$.

8. Rakopoulos C. D. and Hountalas D. T. Development of a new 3-D multi-zone model for indirect injection diesel engines with a swirl type prechamber. Trans. SAE J. Engines, 109 (2000), p. 718-733.

9. Woschni G., Kolesa K., Spindlier W. Isolierung der Brennraumwande-Ein lohendes Enwiklungsziel bei Verbrennundsmotoren. MTZ, 12 1986, p. 495-498.

10. Lebedev S. V., Nechaev L. V. Perfection of parameters of high revolution diesel engines of the unified standard type / Academy of Transport of Russian Federation, AltGTU ). Barnaul: AltGTU, 1999. 112 p.

11. Lebedev S. V. and etc. Formation of constructive range of pistons for high revolution power diesel type. Lebedev S. V., Lebedeva G. V., Matievskiy D. D., Resetov V. I. Under. Red. of D. D. Matievskiy. Academy of Transport of Russian Federation, AltGTU, Barnaul: AltGTU, 2003. 112 p. 